\title{
Comportamientos proactivos en el trabajo: adaptación y análisis psicométrico de una escala
}

\author{
Proactive Work Behaviours: Adaptation and \\ Psychometric Analysis of a Measure
}

\author{
Solana Magalí Salessi ${ }^{1}$ \\ Alicia Omar ${ }^{2}$ \\ Universidad Nacional de Rosario, Argentina \\ Consejo Nacional de Investigaciones Científicas y Técnicas, Argentina
}

Resumen.Objetivo: adaptar la Escala de Comportamientos Proactivos de Belschak y Den Hartog, y analizar sus propiedades psicométricas. Método: se realizó un estudio instrumental sobre una muestra no probabilística de 461 trabajadores argentinos. Resultados: análisis factoriales indicaron la pertinencia de un modelo oblicuo de tres factores $\left(\mathrm{SB} \chi^{2}=1.79 ; \mathrm{GFI}=.95 ; \mathrm{CFI}=.94 ; \mathrm{RMSEA}=.03\right)$ rotulados como comportamientos proactivos orientados a la organización, a los demás, y a uno mismo, respectivamente; con 10 variables observables, invariante según sexo y con adecuada confiabilidad $(\alpha=.78 ; C R=.77 ; \omega=.72)$. Se obtuvo evidencia de validez convergentediscriminante y concurrente a partir del cómputo del índice AVE (AVE $>.50 ; \sqrt{ }$ AVE $<\mathrm{r}_{\mathrm{s}}$ ), y de sus correlaciones con medidas de personalidad proactiva (CP orientados a la organización: $\mathrm{r}_{\mathrm{s}}=.34, p<.01$; CP orientados a los demás: $\mathrm{r}_{\mathrm{s}}=.30, p<.01 ; \mathrm{CP}$ orientados a uno mismo: $\mathrm{r}_{\mathrm{s}}=.36, p<.01$ ), satisfacción (CP orientados a la organización: $\mathrm{r}_{\mathrm{s}}=.40, p<.01 ; \mathrm{CP}$ orientados a los demás: $\mathrm{r}_{\mathrm{s}}=31, p<.01 ; \mathrm{CP}$ orientados a uno mismo: $\mathrm{r}_{\mathrm{s}}=.44$, $p<.01)$ e implicación laboral (CP orientados a la organización: $\mathrm{r}_{\mathrm{s}}=.36, p<.01$; CP orientados a los demás: $\mathrm{r}_{\mathrm{s}}=$ $.25, p<.05$; CP orientados a uno mismo: $\mathrm{r}_{\mathrm{s}}=.42, p<.01$ ). Conclusión: se han obtenido evidencias de validez, invarianza factorial y confiabilidad de la versión argentina del instrumento.

Palabras clave. Proactividad, comportamientos auto-motivados, trabajadores, instrumento de medida, análisis factorial.

Abstract. Objective: To adapt the Belschak \& Den Hartog Proactive Behaviours Scale and analyze their psychometric properties. Method: An instrumental study was carried out on a non-probabilistic sample of 461 Argentine workers. Results: Factorial analysis indicated an oblique model of three factors $\left(\mathrm{SB} \chi^{2}=1.79\right.$; GFI $=.95 ; \mathrm{CFI}=.94 ; \mathrm{RMSEA}=.03)$ labeled as proactive behaviours pro-organizational, prosocial and pro-self, respectively, with 10 observable variables, invariant across to sex and with adequate composite reliability $(\alpha=$ .78; $C R=.77 ; \omega=.72)$. Evidence of convergent, discriminate and concurrent validity was obtained from the calculation of the AVE index (AVE $>.50 ; \sqrt{ } \mathrm{AVE}<\mathrm{r}_{\mathrm{s}}$ ) and its correlations with measures of proactive personality (pro-organizational proactivity: $\mathrm{r} s=.34, p<.01$; prosocial proactivity: $\mathrm{r}_{\mathrm{s}}=.30, p<.01$; pro-self proactivity: $\mathrm{r}$ $=.36, p<.01$ ), job satisfaction (pro-organizational proactivity: $\mathrm{r}_{\mathrm{s}}=.40, p<.01$; prosocial proactivity: $\mathrm{r}_{\mathrm{s}}=31$, $p<.01$; pro-self proactivity: $\mathrm{r}_{\mathrm{s}}=.44, p<.01$ ) and job involvement (pro-organizational proactivity: $\mathrm{r}_{\mathrm{s}}=.36, p<$ .01 ; prosocial proactivity: $\mathrm{r}_{\mathrm{s}}=.25, p<.05$; pro-self proactivity: $\left.\mathrm{r}_{\mathrm{s}}=.42, p<.01\right)$. Conclusion: evidence of validity, factor invariance and reliability of the Argentine version of the scale were obtained.

Keywords. Proactivity, self-starting behaviours, workers, measuring instrument, factorial analysis.

${ }^{1}$ Solana Magalí Salessi. Universidad Nacional de Rosario, Argentina, Consejo Nacional de Investigaciones Científicas y Técnicas. Argentina. Dirección Postal: Ins. de Investigaciones de la Fac. de Hum. y Artes: Entre Ríos 758, CP (2000), Rosario, Argentina. E-mail: solanasalessi@uca.edu.ar

${ }^{2}$ Alicia Omar. Universidad Nacional de Rosario, Argentina, Consejo Nacional de Investigaciones Científicas y Técnicas. Argentina. E-mail: agraomar@yahoo.com

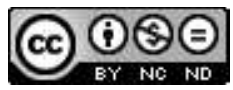

Esta obra está bajo una licencia de Creative Commons Reconocimiento-NoComercial-SinObraDerivada 4.0 Internacional. 


\section{Introducción}

El término proactividad hace referencia a un conjunto amplio y diverso de conductas auto-motivadas y orientadas al cambio, que pretenden influir en el ambiente con el fin de lograr ciertos objetivos individuales, grupales u organizacionales (Belschak \& Den Hartog, 2017; Fatimah, Ferris, \& Frese, 2016; Grant \& Ashford, 2008; Parker \& Collins, 2010). Si bien las primeras publicaciones sobre la temática se remontan al último cuarto del siglo XX (Ashford \& Cummings, 1985; Bateman \& Crant, 1993; Frese, Fay, Hilburger, Leng, \& Tag, 1997), solo recientemente la proactividad se ha posicionado como un tópico central para los estudiosos del comportamiento organizacional. Este renovado interés quizás se deba al reconocimiento de que, además de erigirse en una fuente de mayor satisfacción y compromiso, el comportamiento proactivo puede contribuir a la competitividad organizacional (Thomas, Whitman, \& Viswesvaran, 2010; Tornau \& Frese, 2013).

En el estudio de la proactividad se identifican dos tendencias. Por un lado, una perspectiva de rasgo; por otro lado, un enfoque de corte situacional o interaccionista. Mientras la primera entiende a la proactividad como una disposición endógena estable y permanente (Bateman \& Crant, 1993; Frese et al., 1997); la segunda la concibe como un modo de comportarse que varía en función de las diversas circunstancias y contextos (Belschak \& Den Hartog, 2017; Parker \& Bindl, 2017). La investigación contemporánea se encuentra enmarcada en esta segunda perspectiva, argumentando que, conceptualizar a la proactividad en los términos de rasgo, ofrece muy poca información respecto a qué comportamientos deberían ser considerados "proactivos". Asimismo, en la literatura actual la denominada personalidad proactiva (Bateman \& Crant, 1993) es considerada tan solo uno de los componentes de una constelación más amplia de constructos relacionados a la proactividad (Crant, Hu, \& Jiang, 2017; Parker \& Collins, 2010; Thomas et al., 2010; Tornau \& Frese, 2013).

Si bien podría presuponerse que la proactividad se reduce, exclusivamente, a conductas extra-rol, los especialistas (Belschak \& Den Hartog, 2017; Fatimah et al., 2016; Grant \& Ashford, 2008; Parker \& Collins, 2010) coinciden en señalar que, todos los tipos de desempeño, sean ejecuciones inherentes al rol o acciones que trascienden sus requerimientos formales, pueden ser más o menos proactivos.

En efecto, los criterios actuales para identificar a una conducta como tal son: (1) que sea un comportamiento voluntario basado en motivaciones intrínsecas antes que una acción requerida o coaccionada; (2) que sea una acción centrada en el futuro, es decir, relacionada con la anticipación de oportunidades o problemas; (3) y finalmente, que sea una conducta orientada a producir cambios en uno mismo, en el entorno o en los demás (Belschak \& Den Hartog, 2017; Fatimah et al., 2016; Parker \& Bindl; 2017; Tornau \& Frese, 2013).

La investigación desarrollada a lo largo de estos treinta años ha proporcionado numerosas evidencias de las diferentes formas que puede asumir el comportamiento (Ashford \& Cummings, 1985; Frese et al., 1997; Morrison \& Phelps, 1999). No obstante, Parker y Collins (2010) han demostrado que todas ellas pueden ser subsumidas en tres categorías 
de orden superior; a saber: (a) comportamientos proactivos de ajuste persona-ambiente, que abarcan acciones tendientes a lograr una mayor compatibilidad entre los propios atributos y el entorno organizacional (búsqueda de retroalimentación, negociación de tareas, incitativa personal, etc.); (b) comportamientos laborales proactivos, que engloban los esfuerzos orientados a introducir mejoras en el entorno de trabajo inmediato (toma de control, comportamientos de voz, prevención de problemas, etc.) y; (c) comportamientos estratégicos proactivos, que incluyen acciones orientadas a promover la adaptación de la organización al sistema del cual forma parte (por ejemplo, persuasión sobre temas estratégicos y exploración del mercado en busca de oportunidades o amenazas).

En la misma línea, otros autores (Belschak \& Den Hartog, 2010; 2017; Grant \& Ashford, 2008) han propuesto que los comportamientos proactivos pueden ser clasificados en función de si se dirigen a objetivos individuales, grupales u organizacionales. Estableciendo así las categorías de: (a) proactividad pro-individual, centrada en mejorar el propio desempeño del empleado; (b) proactividad prosocial, direccionada a potenciar el funcionamiento de los compañeros y, a través de ellos, del equipo; y (c) proactividad pro-organizacional, focalizada en contribuir al logro de las metas organizacionales. En el marco de esta conceptualización, Belschak y Den Hartog (2010) han desarrollado un instrumento de medida: la Escala de Comportamientos Proactivos.

La validación inicial fue realizada sobre una muestra de trabajadores holandeses pertenecientes a 86 empresas e industrias de diversos rubros. El conjunto preliminar de ítems se elaboró a partir de medidas preexistentes de iniciativa personal (Frese et al., 1997), proactividad hacia las organizaciones (Griffin, Neal, \& Parker, 2007) y comportamientos de ayuda (Podsakoff, MacKenzie, Paine, \& Bachrach, 2000). Si bien estudios previos (Belschak \& Den Hartog, 2010; 2017; Hwang, Han, \& Chiu, 2015) han reportado evidencias de validez y confiabilidad de este instrumento, hasta el momento no se dispone de una versión adaptada para poblaciones hispanoparlantes. Con miras a cubrir ese vacío empírico-instrumental, el presente estudio se propuso: (a) adaptar la Escala de Comportamientos Proactivos (Belschak \& Den Hartog, 2010) para su empleo en el ámbito laboral argentino, y; (b) analizar las propiedades psicométricas del instrumento.

\section{Método}

\section{Diseño}

El presente estudio se inscribe en la categoría de investigaciones instrumentales (Ato, López, \& Benavente, 2013). Su ejecución se llevó a cabo, de acuerdo con los lineamientos éticos establecidos por la Asociación Americana de Psicología y las recomendaciones del CONICET para las investigaciones en las ciencias sociales y humanas (Resolución 2827/06).

\section{Participantes}

Se integró una muestra inicial de 510 participantes, de los cuales fueron descartados 36 casos incompletos. La muestra definitiva quedó conformada por 474 trabajadores (68\% mujeres) de diversas organizaciones argentinas públicas y privadas localizadas en el centrosur de la provincia de Santa Fe y norte de la provincia de Buenos Aires. El promedio de 
edad fue de 36 años $(D E=10.84)$. La antigüedad laboral media fue de 9 años $(D E=$ 10.21). El 86.5\% de la muestra tenía estudios superiores terciarios y/ o universitarios. El $69 \%$ de los trabajadores tenía un trabajo efectivo, en tanto que el $31 \%$ un contrato de duración limitada. En promedio, los empleados trabajaban 42 horas y media por semana. El 56.7\% trabajaba en el sector privado. La distribución por rubros de actividad fue la siguiente: $32.2 \%$ industria, $30.5 \%$ comercio y servicios, $25.3 \%$ salud, $12 \%$ educación.

\section{Procedimiento}

La recolección de los datos se llevó a cabo en el seno de las organizaciones que aceptaron participar en la investigación, en días y horarios previamente pautados con las autoridades y en los lugares físicos que las mismas dispusieron a tal efecto. La participación de los trabajadores fue voluntaria, anónima y confidencial. No se brindaron incentivos de ningún tipo. La administración de las técnicas estuvo a cargo de personal entrenado a tal efecto. En todos los casos, los participantes completaron de manera individual un cuadernillo que contenía en su primera hoja el objetivo del estudio y las instrucciones para responder; en la segunda hoja, el formulario de consentimiento informado y; en las restantes páginas los ítems correspondientes a los instrumentos que a continuación se describen, distribuidos aleatoriamente.

\section{Instrumentos}

Escala de Comportamientos Proactivos. Se utilizó la escala desarrollada por Belschak y Den Hartog (2010). El instrumento está integrado por 11 ítems presentados en formato Likert de 5 puntos $(1=$ totalmente en desacuerdo; $5=$ totalmente de acuerdo). Los reactivos se distribuyen a lo largo de tres sub-escalas denominadas: (a) comportamientos proactivos pro-organizacionales (3 ítems; ej.: "sugiero ideas frente a los problemas que afronta mi organización"; $\alpha=.82$ ); (b) comportamientos proactivos pro-sociales (4 ítems; ej.: "ayudo a mis compañeros para que desarrollen e implementen nuevas ideas"; $\alpha=.86$ ) y; (c) comportamientos proactivos pro-individuales (4 ítems; ej: "encuentro nuevas maneras de realizar mis tareas y ser más efectivo"; $\alpha=.89)$.

Escala de Personalidad Proactiva. Se administró la versión breve de la escala homónima elaborada por Bateman y Crant (1993). El instrumento está integrado por 10 ítems (ej.:"siempre estoy buscando formas de hacer mejor las cosas") valorados sobre una escala tipo Likert de 5 puntos $(1=$ totalmente en desacuerdo; $5=$ totalmente de acuerdo). Los ítems fueron traducidos del inglés al español por parte de dos jueces expertos. Se revisaron estas versiones hasta obtener una versión consensuada, con especial énfasis en aspectos lingüísticos y culturales. La versión utilizada en este estudio presentó evidencias de confiabilidad compuesta $(C R=.78)$ y consistencia interna $(\alpha=$ .80). El AFC ejecutado mostró que los datos se ajustaban apropiadamente a un modelo uni-factorial integrado por 10 variables observables $\left(\mathrm{S}-\mathrm{B} \chi^{2} / g l=1.71\right.$; GFI $=.95$; $\mathrm{CFI}=$ .94 ; RMSEA $=.03)$.

Escala de satisfacción laboral. Se utilizó la Escala de Satisfacción Laboral Genérica (Salessi \& Omar, 2016). El instrumento está integrado por siete ítems (ej.: "en mi trabajo puedo aplicar todas mis capacidades y habilidades"; $\alpha=.87$ ) valorados sobre una escala tipo Likert de 5 puntos $(1=$ totalmente en desacuerdo; $5=$ totalmente de acuerdo). 
Escala de Implicación con el trabajo. Se empleó la escala desarrollada por Kanungo (1982). $\mathrm{El}$ instrumento está integrado por 10 ítems (ej.: "estoy personalmente comprometido con mi trabajo") valorados sobre una escala tipo Likert de 5 puntos $(1=$ totalmente en desacuerdo; 5 = totalmente de acuerdo). Los ítems fueron traducidos del inglés al español por parte de dos jueces expertos. Se revisaron estas versiones hasta obtener una versión consensuada, con especial énfasis en aspectos lingüísticos y culturales. La versión utilizada en este estudio presentó evidencias de confiabilidad compuesta $(C R=$ .78) y consistencia interna $(\alpha=.81)$. El AFC ejecutado indicó que los datos se ajustaban apropiadamente a un modelo uni-factorial integrado por 10 variables observables (S$\left.\mathrm{B} \chi^{2} / g l=1.96 ; \mathrm{GFI}=.92 ; \mathrm{CFI}=.95 ; \mathrm{RMSEA}=.01\right)$.

\section{Estrategia de análisis}

El procesamiento y análisis de datos se realizó con los programas Factor, SPSS (versión 22.0) y EQS (versión 6.3). La estrategia adoptada para el análisis se detalla a continuación.

Preparación del instrumento. Se analizaron las evidencias de validez de constructo referidas al contenido, la equivalencia semántica y la equivalencia operacional entre el instrumento original y su versión adaptada (Muñiz, Elosua \& Hambleton, 2013). Se efectuaron tareas de revisión bibliográfica y consultas con expertos para establecer el significado del constructo "proactividad", tanto en la cultura donde fue desarrollado el instrumento, como en la cultura objetivo. Se realizó una traducción de los ítems siguiendo un procedimiento iterativo de traducción y re-traducción. En este sentido, dos traductores profesionales tradujeron los ítems desde su idioma original al idioma de la población objetivo. Seguidamente, otros dos profesionales bilingües retradujeron los ítems desde el español al inglés. Finalmente, dos nuevos traductores científicos compararon "a ciegas" las dos formas del instrumento. Con miras a establecer la equivalencia operacional, la versión prototípica fue aplicada a una muestra por disponibilidad de 42 estudiantes de un postgrado en administración de empresas quienes, además, trabajaban en diversas organizaciones ( $55 \%$ varones; edad media: 34 años, $D E=7.64$; antigüedad laboral media: 5.34 años, $D E=3.24)$. Se explicitó la naturaleza y objetivos del estudio, solicitando la colaboración voluntaria de los alumnos. Al finalizar, se habilitó un espacio para que los participantes pudieran opinar sobre el instrumento.

Análisis factoriales. Sobre una mitad de la muestra se ejecutó un análisis factorial exploratorio (AFE), previa obtención de los índices de adecuación muestral (pruebas de Kaiser-Meyer-Olkin y de esfericidad de Bartlett). Por la naturaleza ordinal de los datos se utilizó la matriz de correlaciones policóricas y el método de mínimos cuadrados no ponderados (Hoffmann, Stover, De la Iglesia, \& Fernández-Liporace, 2013). Para determinar el número de factores, se ejecutó un análisis paralelo optimizado, extrayendo aleatoriamente 500 sub-matrices e implementando el análisis de rango mínimo. Se efectuó la extracción de los factores sugeridos, optando por la rotación oblicua Promin, ya que existía la presunción que los elementos de la escala estuvieran relacionados (Baglin, 2014). El criterio para la selección de los ítems fue que pesaran .40 o más sobre el factor, y que no saturaran sobre más de un factor al mismo tiempo (Lloret-Segura, Ferreres, Hernández, \& Tomás, 2014). 
Sobre la otra mitad de la muestra se llevó a cabo un análisis factorial confirmatorio (AFC). Se optó por la estrategia de modelos rivales (Hair, Black, Babin, Anderson, \& Tatham, 2010), contrastando el modelo derivado del AFE con un modelo alternativo uni-factorial sugerido por la literatura (Kamia \& Porto, 2009). Para estimar cada modelo se utilizó el método de máxima verosimilitud con la corrección robusta de Satorra-Bentler (S-B), dada la naturaleza ordinal de los datos (Bentler, 2006). Para evaluar la bondad de ajuste se analizó que: (a) el índice $\mathrm{S}-\mathrm{B} \chi$ sobre los grados de libertad $\left(\mathrm{S}-\mathrm{B} \chi^{2} / g \emptyset\right.$ fuera inferior a 3; (b) que el índice de bondad de ajuste (GFI, Goodness of Fit Index) y el índice de ajuste comparativo (CFI, Comparative Fit Index) alcanzaran valores iguales o superiores a .90; y (c) que el error cuadrático medio de aproximación (RMSEA, Root Mean Square Error of Approximation) fuera inferior a .05. Asimismo, se examinó el Criterio de Información de Akaike (AIC, Akaike Information Criterion), a sabiendas que cuanto menor sea su valor, más parsimonioso es el modelo (Hair et al., 2010).

Análisis de invarianza factorial. Dada la desproporción en la representación de ambos sexos en la muestra se procedió a realizar un análisis de invarianza según sexo. Específicamente, se examinó la invarianza configural (referente a la estructura factorial) y la invarianza de medida estricta (relativa a las cargas factoriales, varianzas, covarianzas y varianza residual). Para esto, inicialmente se estimó un modelo de base sin restricciones para ambos grupos. En segundo lugar, se propusieron modelos alternativos con restricciones en los diversos parámetros. Para la comparación de los modelos anidados se utilizó la prueba de diferencia de $\chi^{2}$, a sabiendas que si es estadísticamente significativa, los mismos no son equivalentes entre los grupos (Dimitrov, 2010).

Análisis de validez. Se obtuvo evidencia de validez convergente, discriminante y concurrente. La validez convergente y discriminante se determinaron mediante el cálculo de la varianza media extraída (AVE, Average Variance Extracted) y de su raíz cuadrada, respectivamente (Bagozzi \& Yi, 2012; Henseler, Ringle, \& Sarstedt, 2015). El AVE permite estimar la varianza común entre los indicadores y su factor latente, considerando que valores superiores a .50 indican que más del $50 \%$ de la varianza del constructo es debida a sus indicadores. Por su parte, valores de la raíz cuadrada del AVE superiores a la correlación entre los factores latentes demuestran que cada constructo comparte más varianza con sus indicadores que con los demás. La validez concurrente se analizó a partir del cálculo de las correlaciones entre los constructos estudiados, mediante el cómputo del coeficiente de rho Spearman.

Análisis de confiabilidad. Las evidencias de confiabilidad se establecieron a partir del cómputo del coeficiente alfa ordinal, del coeficiente de confiabilidad compuesta (CR, Composite Reliability) y del coeficiente Omega de McDonald ( $\omega$ ) con sus respectivos intervalos de confianza. Valores de alfa y CR por encima de .70 se consideran evidencia de adecuada confiabilidad (Gadermann, Guhn \& Zumbo, 2012; Hair et al., 2010). El coeficiente $\omega$ es un estimador de consistencia interna basado en las cargas factoriales, que indica la proporción de varianza atribuida a la totalidad de la varianza común. Valores entre .70 y .90 se consideran aceptables (Ventura-León \& Caycho, 2017). 


\section{Resultados}

\section{Preparación del instrumento}

$\mathrm{El}$ análisis de la literatura permitió concluir que el constructo proactividad es equivalente en ambas culturas (Salessi \& Omar, 2017). Los profesionales indicaron que se había mantenido inalterada la concordancia entre cada ítem original y el traducido al español. Con respecto a la equivalencia operacional, los participantes del estudio piloto señalaron que las instrucciones para realizar la tarea estaban claramente redactadas; que no tuvieron inconvenientes para comprender el contenido de los ítems y que la escala tipo Likert utilizada para responder no generaba dificultades.

\section{Análisis exploratorios}

El porcentaje de datos perdidos en cada ítem no superó el 5\%, respondiendo a una distribución aleatoria. Los valores faltantes fueron reemplazados mediante el método de Estimación-Maximización (Tabachnick \& Fidell, 2013). Se detectaron 13 casos atípicos, los que fueron excluidos de la matriz de datos. La muestra final quedó, entonces, constituida por 461 participantes. Excepto el ítem 10 (que mostró valores de curtosis superiores a 1.5), en el resto de los ítems los índices de asimetría y curtosis se encontraron dentro del rango esperado. Las correlaciones ítem-total fueron todas positivas. La tabla 1 presenta los estadísticos descriptivos, índices de asimetría y curtosis e índices de discriminación correspondientes.

Tabla 1

Estadísticos descriptivos, indices de asimetría y curtosis y correlación item-total corregida correspondientes a los items de la adaptación argentina de la Escala de Comportamientos Proactivos en el Trabajo

\begin{tabular}{cccccc}
\hline ÍTEM & Media & $D E$ & Asimetría & Curtosis & $r$ i-total \\
\hline 1 & 3.02 & 1.05 & -.73 & -1.36 & .55 \\
2 & 3.81 & 1.10 & -.88 & .94 & .50 \\
3 & 3.32 & 1.13 & .47 & .81 & .54 \\
4 & 3.31 & 1.26 & -.31 & -.91 & .67 \\
5 & 3.46 & 1.00 & -.92 & 1.07 & .65 \\
6 & 3.48 & 1.17 & -.72 & .77 & .63 \\
7 & 3.04 & 1.22 & .80 & -.74 & .72 \\
8 & 3.47 & 1.06 & .65 & -.95 & .61 \\
9 & 3.30 & 1.11 & .93 & -.99 & .68 \\
10 & 3.24 & 1.12 & -1.38 & -1.81 & .40 \\
11 & 3.65 & 1.04 & -.85 & .73 & .65 \\
\hline
\end{tabular}




\section{Análisis factoriales}

Atendiendo a la recomendación de contar con un mínimo de 200 observaciones para asegurar que la solución factorial fuera estable y generalizable (Lloret-Segura et al., 2014), se seleccionaron en forma aleatoria simple 231 casos $(n=461)$. La matriz de datos fue considerada factorizable (Test de esfericidad de Bartlett: $\chi^{2}(36 ; 231)=560.82, p<$ .001; Índice de adecuación muestral de Kaiser-Meyer-Olkin $=.80)$. El análisis paralelo optimizado, sugirió tres factores con autovalores superiores a sus equivalentes de la matriz de datos aleatorios. El porcentaje global de varianza común explicada por los factores extraídos fue de $68.70 \%$. La distribución de los reactivos coincidió con las dimensiones propuestas originalmente por Belschak y Den Hartog (2010), las que fueron rotuladas como comportamientos proactivos orientados a la organización, comportamientos proactivos orientados a los demás y comportamientos proactivos orientados a uno mismo. En la tabla 2 se informa, tanto la matriz de configuración con las saturaciones factoriales de cada ítem en cada factor, como la matriz de estructura que contiene las correlaciones entre las variables observables y las variables latentes.

Sobre la otra mitad de la muestra $(n=230)$ se llevó a cabo un análisis factorial confirmatorio siguiendo los lineamientos de la estrategia de modelos rivales (Kline, 2011; Bentler, 2006). Los resultados obtenidos para un modelo uni-factorial (un factor general de proactividad con 11 ítems como variables observables y sus respectivos errores de medida) indicaron un ajuste inadecuado $\left(\mathrm{SB} \chi^{2}=3.41\right.$; GFI $=.80$; $\mathrm{CFI}=.77$; RMSEA $=$ .11 , IC $95 \%$ [0.10; 0.14]; AIC = 194.33). Las cargas factoriales se ubicaron entre .30 y .65 estandarizadas $(\phi<.05)$. La eliminación de los ítems con menores cargas (ítem 6: "oriento a los nuevos compañeros" e ítem 10: "me esfuerzo por lograr mis objetivos personales en mi trabajo") no produjo una mejora significativa en el ajuste $\left(\mathrm{SB} \chi^{2}=2.94\right.$; $\mathrm{GFI}=.82$; $\mathrm{CFI}=.80 ;$ RMSEA $=.10$, IC 95\% [.09; .12]; AIC = 164.89). La inspección de los índices de modificación, permitió observar que un cambio en los parámetros estimados entre los errores pertenecientes al par conformado por el ítem 3 ("optimizo la distribución de mi trabajo para ayudar a que la organización logre sus metas") e ítem 8 ("busco nuevos modos de realizar mi trabajo para ser más eficiente") produce una ganancia en el modelo. No obstante, tampoco fue suficiente para alcanzar un ajuste satisfactorio $\left(\mathrm{SB} \chi^{2}=2.11\right.$; $\mathrm{GFI}=.84 ; \mathrm{CFI}=.82 ; \mathrm{RMSEA}=.09, \mathrm{IC} 95 \%[.08 ; .11] ; \mathrm{AIC}=151.29)$.

Por su parte, los resultados obtenidos para un modelo tri-factorial (3 factores oblicuos, con 11 ítems como indicadores observables y sus respectivos errores de medida) indicaron un grado de ajuste aceptable pero no óptimo $\left(\mathrm{SB} \chi^{2}=2.79 ; \mathrm{GFI}=.85 ; \mathrm{CFI}=.81\right.$; RMSEA $=.04$, IC $95 \%[.03 ; .05] ;$ AIC $=143.91)$. Las cargas factoriales se ubicaron entre .42 y .79 estandarizadas $(\not<.05)$. La eliminación del ítem con menor carga factorial (ítems 10: "me esfuerzo por lograr mis objetivos personales en mi trabajo") produjo una leve mejora en el ajuste $\left(\mathrm{SB} \chi^{2}=2.36\right.$; $\mathrm{GFI}=.88$; $\mathrm{CFI}=.86$; RMSEA $=.04, \mathrm{IC} 95 \%[.03 ; .05]$; $\mathrm{AIC}=$ 123.18). La inspección de los índices de modificación permitió observar que un cambio en los parámetros estimados entre los errores pertenecientes al par conformado por el ítem 9 ("me capacito para desarrollarme profesionalmente") e ítem 11 ("asumo tareas que contribuyen al desarrollo de mi carrera laboral") produce una ganancia en el modelo $\left(\mathrm{SB} \chi^{2}=1.79 ; \mathrm{GFI}=.95 ; \mathrm{CFI}=.94 ; \mathrm{RMSEA}=.03, \mathrm{IC} 95 \%\right.$ [.04; .05]; $\left.\mathrm{AIC}=112.68\right)$. Los 
Tabla 2

Matriz de estructura y matriz de configuración correspondiente a los items de la adaptación argentina de la Escala de Comportamientos Proactivos en el Trabajo

\begin{tabular}{|c|c|c|c|c|c|c|}
\hline \multirow[t]{2}{*}{ Dimensiones y contenido del ítem } & \multicolumn{3}{|c|}{$\begin{array}{c}\text { Matriz de } \\
\text { Configuración }\end{array}$} & \multicolumn{3}{|c|}{$\begin{array}{l}\text { Matriz de } \\
\text { estructura }\end{array}$} \\
\hline & I & II & III & I & II & III \\
\hline \multicolumn{7}{|l|}{ Comportamientos proactivos orientados a la organización } \\
\hline 1. Sugiero soluciones para los problemas de mi organización & .73 & & & .80 & & \\
\hline $\begin{array}{l}\text { 2. Aprendo nuevos conocimientos que beneficiarán a mi } \\
\text { organización }\end{array}$ & .68 & & & .66 & & \\
\hline $\begin{array}{l}\text { 3. Optimizo la distribución de mi trabajo para ayudar a que la } \\
\text { organización logre sus metas }\end{array}$ & .72 & & & .93 & & \\
\hline \multicolumn{7}{|l|}{ Porcentaje de varianza explicada: $26.81 \%$} \\
\hline \multicolumn{7}{|l|}{ Comportamientos proactivos orientados a los demás } \\
\hline 4. Comparto mis conocimientos con mis compañeros & & .75 & & & .81 & \\
\hline $\begin{array}{l}\text { 5. Colaboro con las tareas de mis compañeros, aunque no esté } \\
\text { obligado a hacerlo }\end{array}$ & & .72 & & & .78 & \\
\hline 6. Oriento a los nuevos compañeros & & .78 & & & .80 & \\
\hline 7. Ayudo a que mis compañeros concreten sus ideas & & .76 & & & .87 & \\
\hline \multicolumn{7}{|l|}{ Porcentaje de varianza explicada: $22,19 \%$} \\
\hline \multicolumn{7}{|l|}{ Comportamientos proactivos orientados a uno mismo } \\
\hline $\begin{array}{l}\text { 8. Busco nuevos modos de realizar mi trabajo para ser más } \\
\text { eficiente }\end{array}$ & & & .80 & & & .81 \\
\hline 9. Me capacito para desarrollarme profesionalmente & & & .86 & & & .85 \\
\hline $\begin{array}{l}\text { 10. Me esfuerzo por lograr mis objetivos personales en mi } \\
\text { trabajo }\end{array}$ & & & .42 & & & .45 \\
\hline $\begin{array}{l}\text { 11. Asumo tareas que contribuyen al desarrollo de mi carrera } \\
\text { laboral }\end{array}$ & & & .85 & & & .80 \\
\hline Porcentaje de varianza explicada: $18.98 \%$ & & & & & & \\
\hline
\end{tabular}

tres factores correlacionaron positiva y significativamente, con coeficientes variando entre .47 y $.59(p<.001)$. Las cargas factoriales estandarizadas en el factor comportamientos proactivos orientados a la organización se ubicaron entre .56 y $.68(p<.05)$; entre .54 y .66 $(p<.05)$ en el factor comportamientos proactivos orientados a los demás y; entre 48 y .75 $(\phi<.05)$ en el factor comportamientos proactivos orientados a uno mismo.

Análisis de invarianza factorial

El modelo de base estuvo compuesto por tres factores latentes oblicuos, 10 ítems como variables observables y sus respectivos errores de medición. Los índices de ajuste obtenidos por este modelo fueron adecuados $\left(\mathrm{S}-\mathrm{B} \chi^{2}=218.01, \mathrm{GFI}=.91, \mathrm{CFI}=.90\right.$, $\mathrm{RMSEA}=$ 
.03). Similares valores de ajuste se observaron para el modelo con restricciones en los pesos factoriales $\left(\mathrm{S}-\mathrm{B} \chi^{2}=218.88, \mathrm{GFI}=.90, \mathrm{CFI}=.89\right.$, RMSEA $\left.=.04\right)$; con restricciones en las varianza y covarianza $\left(\mathrm{S}-\mathrm{B} \chi^{2}=239.91, \mathrm{GFI}=.89, \mathrm{CFI}=.89, \mathrm{RMSEA}=.04\right)$ y con restricciones en la varianza residual de los factores $\left(\mathrm{S}-\mathrm{B} \chi^{2}=247.27, \mathrm{GFI}=.88, \mathrm{CFI}=.87\right.$, RMSEA .05). En todos los casos, la diferencia entre los respectivos $\chi^{2}$ entre el modelo de base y los modelos con restricciones no fue estadísticamente significativo (D $\chi^{2}=.80, \mathrm{gl}$ $=4, p=.93 ; \mathrm{D} \chi^{2}=21.9, g l=18, p=.23 ; \mathrm{D} \chi^{2}=21,1, g l=23, p=.18$, respectivamente), demostrando la invarianza de la escala entre varones y mujeres.

Análisis de validez convergente, discriminante y concurrente

La tabla 3 muestra los coeficientes de correlación y los valores correspondientes al índice AVE y a su raíz cuadrada. Se observa que todos los comportamientos proactivos se relacionan positivamente a la personalidad proactiva, a la satisfacción laboral y a la implicación con el trabajo; siendo tales asociaciones levemente superiores para los comportamientos proactivos orientados a uno mismo y para los comportamientos proactivos orientados a la organización. Los coeficientes AVE obtenidos evidencian validez convergente, habida cuenta que la varianza capturada por los factores identificados es mayor que la debida a los errores de medida. Asimismo, todos los constructos presentan evidencia de validez discriminante, desde el momento que la raíz cuadrada del AVE es mayor que la correlación al cuadrado entre cada factor y los demás (Bagozzi \& Yi, 2012; Henseler et al., 2015).

\section{Análisis de confiabilidad}

Se obtuvo evidencia de fiabilidad tanto para escala total $(\alpha=.78 ; C R=.77 ; \omega=.72$, IC $95 \%[.69 ; .75]$ como para cada uno de sus sub-escalas. En este sentido, los valores para el factor comportamientos proactivos orientados a la organización se encontraron dentro del rango esperado $(\alpha=.80 ; C R=.79 ; \omega=.74$, IC 95\% $[.72 ; .79])$; mientras que, los valores obtenidos para los factores comportamientos proactivos orientados a los demás y comportamientos proactivos orientados a uno mismo, fueron ligeramente superiores $(\alpha$ $=.82 ; C R=.80 ; \omega=.79$, IC 95\% [.77; 83]; $\alpha=.81 ; C R=.79 ; \omega=.76$, IC 95\% $[.73 ; 81]$, respectivamente).

Tabla 3

Indices de correlación y varianza media extraída correspondientes a las variables bajo estudio

\begin{tabular}{lccccccc}
\multicolumn{1}{c}{ Variables } & 1 & 2 & 3 & 4 & 5 & 6 & AVE \\
\hline 1. CP orientados a la organización & $(.86)$ & $.55^{*}$ & $.48^{*}$ & $.34^{*}$ & $.40^{*}$ & $.36^{*}$ & .74 \\
2. CP orientados a los demás & & $(.87)$ & $.42^{*}$ & $.30^{*}$ & $.31^{*}$ & $.25^{* *}$ & .76 \\
3. CP orientados a uno mismo & & & $(.88)$ & $.36^{*}$ & $.44^{*}$ & $.42^{*}$ & .79 \\
4. Personalidad proactiva & & & & $(.87)$ & $.38^{*}$ & $.30^{*}$ & .77 \\
5. Satisfacción laboral & & & & & $(.92)$ & $.67^{*}$ & .85 \\
6. Implicación laboral & & & & & & $(.84)$ & .72 \\
\hline
\end{tabular}

Nota: Sobre la diagonal se informa la raíz cuadrada del AVE. CP = comportamientos proactivos.

$p<.10 .^{*} p<.05 . * *$ 


\section{Discusión}

La investigación realizada tuvo como objetivos adaptar y validar la Escala de Comportamientos Proactivos en el Trabajo desarrollada por Belschak y Den Hartog (2010). Los análisis factoriales desarrollados indican que, en comparación a un modelo uni-factorial, un modelo oblicuo de tres factores correlacionados es el que mejor se ajusta a la matriz de varianzas y covarianzas analizada. Tales hallazgos armonizan con las conclusiones de los autores del instrumento (Belschak \& Den Hartog, 2010; 2017), así como con la adaptación efectuada por Hwang et al. (2015) sobre una muestra de 259 trabajadores taiwaneses. Sin embargo, a diferencia de estas versiones, en esta adaptación solo se retuvieron 10 ítems como indicadores observables, prescindiendo de uno de los reactivos que integraban la sub-escala comportamientos proactivos orientados a uno mismo, dada su baja saturación en dicho factor.

Paralelamente, los resultados obtenidos proporcionan evidencia favorable a la invarianza factorial del instrumento en relación con el sexo de los participantes. Vale decir que, el modelo de medida sugerido por el AFE y confirmado por el AFC no presenta variaciones significativas entre varones y mujeres. Asimismo, el presente estudio presenta evidencias de validez convergente-discriminante, desde el momento que la varianza capturada por los factores identificados es mayor que la debida a los errores de medida y se puede explicar adecuadamente a través de los indicadores elegidos (Bagozzi \& Yi, 2012; Henseler et al., 2015). Las relaciones entre los tres factores se ubicaron en el orden de .42 a .55, coincidiendo con las asociaciones informadas por Belschak y Den Hartog (2010; 2017). Los índices de confiablidad obtenidos fueron adecuados, encontrándose en línea con los valores informados en otros estudios (Belschak \& Den Hartog, 2017; Hwang, et al., 2015). En conjunto, estos hallazgos proporcionan evidencia favorable a la homogeneidad de los ítems, permitiendo aseverar que las 3 sub-escalas miden de forma consistente la característica para la cual fueron elaboradas. Del mismo modo, se muestran en armonía con la literatura especializada (Belschak \& Den Hartog, 2017; Grant \& Ashford, 2008; Parker \& Collins, 2010); la que señala que los comportamientos proactivos se desglosan en tres categorías diferentes en función de los objetivos (individuales, grupales u organizacionales) que los motivan.

En lo que respecta a la validez concurrente, el análisis de correlaciones indica que los comportamientos proactivos se asocian en la dirección esperada, tanto entre sí como con el resto de los constructos evaluados. Así, por ejemplo, las correlaciones entre comportamientos proactivos y satisfacción laboral coinciden con la literatura publicada (Ismaeli, Nowalid, \& Bakar, 2016; Thomas et al., 2010; Tornau \& Frese, 2013) mostrando, una vez más, que la proactividad se asocia a actitudes positivas hacia el trabajo y hacia las organizaciones. En lo que respecta a la implicación laboral, si bien hasta la fecha son escasos los estudios que se han interesado por examinar sus vinculaciones directas, la evidencia disponible se encuentra en línea con los presentes hallazgos. En este sentido, las conclusiones de Mallin, Ragland y Finkle (2014) subrayan que los trabajadores que desarrollan comportamientos proactivos se encuentran cognitiva y emocionalmente absorbidos y comprometidos por su trabajo, concibiéndolo como un dominio central en sus vidas y un aspecto crítico para su autoestima y autoimagen. Finalmente, las 
asociaciones positivas observadas con personalidad proactiva replican los resultados de múltiples metaanálisis (Crant, et al., 2017; Thomas et al., 2010; Tornau \& Frese, 2013), corroborando la vinculación entre la predisposición a controlar el ambiente (personalidad proactiva) y la posibilidad de manifestar acciones deliberadas orientadas al cambio (comportamientos proactivos).

Entre las principales limitaciones de la investigación realizada hay que citar la representatividad de la muestra; la que, por un lado, presentó un porcentaje superior de mujeres y, por otro lado, fue seleccionada en forma no probabilística. Si bien la generalización de los resultados a la población en su conjunto se ve obstaculizada, vale destacar que, a pesar de la desproporción en el sexo de los participantes, la invarianza factorial de la escala quedó garantizada. Una segunda limitación podría estar vinculada con la validez externa del instrumento aquí presentado. En este sentido, como no se ha verificado la estabilidad de las dimensiones a lo largo del tiempo sería recomendable que próximos estudios exploren la confiabilidad test-retest. Del mismo modo, no haber analizado otros aspectos tales como la validez predictiva e incremental, podría constituir una tercera limitación. Frente a este panorama, se alienta a que otras investigaciones examinen si las puntuaciones en esta escala constituyen predictores válidos y precisos de comportamientos relevantes para las organizaciones.

Por último, cabe remarcar que no haber examinado la posibilidad de que los hallazgos pudiesen estar contaminados por sesgos, tales como los asociados al método común y a la deseabilidad social, también podría considerarse una limitación del presente estudio. Producto de estos fenómenos las relaciones entre las variables de interés se verían indefectiblemente afectadas, desde el momento que tales sesgos son independientes de la varianza sustantiva que pueda existir entre ellas. Así, por ejemplo, si el deseo de "aparecer mejor" influyó las respuestas; entonces, la correlación encontrada obedecería más a este hecho que a su verdadera asociación teórica. Incluso, cabe la posibilidad que variables que no comparten varianza alguna exhiban correlaciones significativas como resultado del método de recolección utilizado. Esto último quizás podría explicar por qué, tanto en este estudio como en investigaciones precedentes (Crant, et al., 2017; Thomas et al., 2010), la personalidad proactiva exhibe asociaciones con medidas que operacionalizan a la proactividad en términos de comportamientos, cuando tales correlaciones deberían ser exiguas (Tornau \& Frese, 2013). Frente a este panorama, se sugiere que futuros estudios correlacionales sobre proactividad que midan simultáneamente los constructos de personalidad proactiva y comportamientos proactivos por medio de una encuesta autoadministrada, analicen la existencia de sesgos debido a la varianza del método común, apelando a algunas de las estrategias desarrolladas a tal efecto (Podsakoff, MacKenzie, \& Podsakoff, 2012). Asimismo, sería importante que pudieran incluir otros mecanismos de validez interna tales como, escalas específicamente desarrolladas para medir la deseabilidad social.

Como corolario de estas reflexiones vale subrayar que, aun cuando la utilización de instrumentos de autoinforme constituye una práctica habitual en la evaluación psicológica por razones pragmáticas, la investigación contemporánea sobre proactividad precisa de métodos de medición consistentes con la concepción situacional vigente. La literatura sobre la temática se encuentra atravesada por una profunda confusión e incongruencia 
entre las definiciones conceptuales y operacionales del constructo. Al respecto, diversos autores (Frese et al., 1997; Parker, Williams, \& Turner, 2006; Torneau \& Frese, 2013) han subrayado que identificar un comportamiento como "proactivo" depende en parte del contexto, por lo que es indispensable desarrollar un enfoque de medición específico que atienda a las particularidades de este.

Del mismo modo, obtener información de otras fuentes, tales como compañeros y supervisores, constituiría una alternativa superadora en la evaluación de los comportamientos proactivos. Así, por ejemplo, las entrevistas podrían constituir un método idóneo, desde el momento que la información del observador se encuentra basada en la observación del comportamiento y no en la personalidad del empleado objeto de la observación; vale decir, en sus sentimientos y pensamientos internos (Frese et al., 1997; Hogan \& Shelton, 1998; Torneau \& Frese, 2013). Además, las evaluaciones situacionales podrían constituir un recurso valioso para la predicción de comportamientos proactivos (Lievens \& Motowidlo, 2016).

En última instancia, la elección del método deberá obedecer a una constelación de factores, tales como el diseño del estudio, el acceso a la muestra, quién se encuentra en la mejor posición para evaluar con precisión la proactividad, etc.

Entre las fortalezas, vale destacar que el trabajo realizado constituye la primera tentativa de operacionalizar el constructo proactividad en Argentina, por lo que representa una contribución genuina para que futuros estudios puedan ser concretados con el recurso de un instrumento respaldado por evidencias de confiabilidad y validez. La validación efectuada abre la necesidad de llevar a cabo nuevos estudios instrumentales en otros contextos laborales y socioculturales. En este sentido, sería necesario presentar evidencias adicionales de las propiedades psicométricas de la escala aquí presentada, analizando para ello los datos proporcionados por otras muestras de trabajadores. Del mismo modo, sería importante desarrollar otras pruebas más adecuadas y eficientes, enmarcadas en modelos más idóneos (por ejemplo, modelos tipo Rasch), capaces de subsanar las limitaciones de las pruebas construidas según la teoría clásica de los tests. Más allá de estas recomendaciones, el instrumento aquí validado queda a partir de este momento, disponible para investigadores y profesionales de recursos humanos.

Contar con una herramienta adaptada a la realidad local conlleva múltiples beneficios prácticos. El más importante quizás, sea la posibilidad de hacer un diagnóstico preliminar del comportamiento proactivo y delinear acciones consecuentes. Al respecto, vale destacar que la investigación contemporánea (Belschak \& Den Hartog, 2017; Fatimah et al., 2016; Grant \& Ashford, 2008; Parker \& Wang, 2015) muestra que la variabilidad interindividual de la proactividad, no se debe tanto a las diferencias en rasgos fijos y estables, sino a características maleables y abiertas al cambio. De aquí que sea lícito sostener que, en mayor o menor medida, todas las personas pueden ser proactivas en diversas circunstancias y; paralelamente, que la proactividad pueda ser desarrollada con intervenciones específicas.

Por lo tanto, las organizaciones preocupadas por incrementar su productividad y garantizar su competitividad, deberían concentrarse en la creación de un entorno capaz de contribuir al florecimiento de comportamientos proactivos. En efecto, la evidencia empírica 
(Caesens, Marique, Hanin, \& Stinglhamber, 2016) señala que aquellos trabajadores que perciben que la organización valora sus contribuciones y se preocupa por su bienestar presentan mayor cantidad de comportamientos proactivos; principalmente del tipo proorganizacionales. Del mismo modo, se ha subrayado (Parker \& Wing, 2015) que las percepciones de apoyo y la confianza en el equipo actúan como facilitadores de este tipo de conductas. Paralelamente, se ha comprobado que un estilo de liderazgo transformacional resulta en mayor proactividad en rol y extra rol (Schmitt, Den Hartog, \& Belschak, 2016). Finalmente, se ha indicado (Maden, 2016) que procesos de trabajos flexibles y puestos enriquecidos por la autonomía y la diversidad de tareas predisponen favorablemente a comportamientos proactivos; posiblemente, dada la mayor libertad, responsabilidad y reto que presuponen para el trabajador.

\section{Referencias}

Ashford, S. J., \& Cummings, L. L. (1985). Proactive feedback seeking: The instrumental use of the information environment. Journal of Occupational Psychology, 58,67-79. doi: 10.1111/j.2044-83251985.tb00181.x

Ato, M., López, J. J., \& Benavente, A. (2013). Un sistema de clasificación de los diseños de investigación en psicología. Anales de Psicología, 29(3), 1038-1059. doi: 10.6018/ analesps.29.3.178511

Baglin, J. (2014). Improving your exploratory factor analysis for ordinal data: A demonstration using FACTOR. Practical Assessment, Research \& Evaluation, 19(5), 2-15. Retrieved February 2015 from http://pareonline.net/getvn. asp?v $=19 \& n=5$

Bagozzi, R. \& Yi, Y. (2012). Specification, evaluation, and interpretation of structural equation models. Journal of the Academy of Marketing Science, 40, 8-34. doi: 10.1007/ s11747-011-0278-x

Bateman, T. S., \& Crant, J. M. (1993). The proactive component of organizationalbehaviour: A measure and correlates. Journal of Organizational Behaviour, 14, 103118. doi: $10.1002 /$ job. 4030140202

Belschak, F., \& Den Hartog, D. (2017). Foci of proactive behaviour. In S. Parker, \& U. Bindl, (Eds.), Proactivity at work: Making things happen in organizations (pp. 169-189). New York, NY: Routledge.

Belschak, F. D., \& Den Hartog, D. N. (2010). Pro-self, prosocial, and pro-organizational foci of proactive behaviour: Differential antecedents and consequences. Journal of Occupational and Organizational Psychology, 83, 475-498. doi: 10.1348/096317909x439208

Bentler, P. (2006). EQS 6 Structural Equations Program Manual. Los Angeles, CA: Multivariate Software Inc.

Caesens, G., Marique, G., Hanin, D., \& Stinglhamber, F. (2016). The relationship between perceived organizational support and proactive behaviour directed towards the 
organization. European Journal of Work \& Organizational Psychology, 25, 398-411. doi: 10.1080/1359432X.2015.1092960

Crant, M. Hu, J., \& Jiang, K. (2017). Proactive personality: A twenty-year review. In S. Parker, \& U. Bindl, (Eds.), Proactivity at work: Making things happen in organizations (pp. 194-225). New York, NY: Routledge

Dimitrov, D. M. (2010). Testing for factorial invariance in the context of construct validation. Measurement and Evaluation in Counselling and Development, 43(2), 121-149. doi: $10.1177 / 0748175610373459$

Fatimah, S., Ferris, L., \& Frese, M. (2016). Proactivity research: New directions and concepts. Academy of Management Annual Meeting Proceedings, 1-1. doi: 10.5465/ AMBPP.2016.12840symposium.

Frese, M., Fay, D., Hilburger, T., Leng, K., \& Tag, A. (1997). The concept of personal initiative: Operationalization, reliability and validity in two German samples. Journal of Occupational \& Organizational Psychology, 70, 139-161. doi: 10.1111/j.204483251997.tb00639.x

Gadermann, A. M., Guhn, M., \& Zumbo, D. (2012). Estimating ordinal reliability for Likert-type and ordinal item response data: A conceptual, empirical, and practical guide. Practical Assessment, Research \& Evaluation, 17(3), 1-13. Retrieved March 2014 from http://pareonline.net/pdf/v17n3.pdf

Grant, A. M., \& Ashford, S. J. (2008). The dynamics of proactivity at work. Research in Organizational Behaviour, 28, 3-34. doi: 10.1016/j.riob.2008.04.002

Griffin, M., Neal, A., \& Parker, S. (2007). A new model of work role performance: Positive behaviour in uncertain and interdependent contexts. Academy of Management Journal, 50, 327-347. doi: 10.5465/AMJ.2007.24634438

Hair, J. E., Black, W. C., Babin, B. J., Anderson, R. E., \& Tatham, R. L. (2010). Multivariate data analysis (6th ed.). Upper Saddle River, NJ: Pearson-Prentice Hall.

Henseler, J., Ringle, C. M., \& Sarstedt, M. (2015). A new criterion for assessing discriminant validity in variance-based structural equation modelling. Journal of the Academy of Marketing Science, 43(1), 115-135. doi: 10.1007/s11747-014-0403-8

Hoffmann, A., Stover, J., De la Iglesia, G., \& Fernández-Liporace, M. (2013). Correlaciones policóricas y tetracóricas en estudios factoriales exploratorios y confirmatorios. Ciencias Psicológicas, 7, 151-164.

Hogan, R., \& Shelton, D. (1998). A socioanalytic perspective on job performance. Human Performance, 11, 129-144. doi: 10.1080/08959285.1998.9668028

Hwang, P., Han, M., \& Chiu, S. (2015). Role breadth self-efficacy and foci of proactive behaviour: Moderating role of collective, relational, and individual self-concept. The Journal of Psychology, 149(8), 846-865. doi: $10.1080 / 00223980.2014 .985284$ 
Ismaeli, A., Nowalid, W., \& Bakar, R. (2016). Proactive behaviour as a mediator of the relationship between career management and career satisfaction. Journal Pengurusan, 48, 1-19.

Kamia, M., \& Porto, J. B. (2009). Desenvolvimento e validação da escala de Comportamento Proativo nas Organizações - ECPO. Avaliação Psicológica, 8, 359-367. doi: 10.1590/ s1414-98932011000300003

Kanungo, R. N. (1982). Measurement of job and work involvement. Journal of Applied Psychology, 67, 341-349. doi: 10.1037/0021-9010.67.3.341

Kline, R. B. (2011). Principles and practice of structural equation modeling. New York, NY: Guilford Press.

Lievens, F., \& Motowidlo, S. (2016). Situational judgment tests: From measures of situational judgment to measures of general domain knowledge. Industrial and Organizational Psychology, 9(1), 3-22. doi:10.1017/iop.2015.71

Lloret-Segura., Ferreres, A., Hernández, A., \& Tomás, I. (2014). El análisis factorial exploratorio de los ítems: una guía práctica, revisada y actualizada. Anales de Psicología, 30(3), 1151-1169. doi: 10.6018/analesps.30.3.199361

Maden, C. (2016). Linking high involvement human resource practices to employee proactivity. Personnel Review, 44, 720-738. doi: 10.1108/PR-01-2014-0030

Mallin, M., Ragland, C., \& Finkle, T. (2014). The proactive behaviour of youngest salespeople: Antecedents and outcomes. Journal of Marketing Channels, 21(4), 268278. doi: 10.1080/1046669X.2014.945359

Morrison, E. \& Phelps, C. (1999). Taking charge at work: Extra-role efforts to initiate workplace change. The Academy of Management Journal, 42, 403-419. doi: $10.2307 / 25701$

Muñiz, J., Elosua, P., \& Hambleton, R. (2013). Directrices para la traducción y adaptación de los tests: segunda edición. Psicothema, 25, 151-157. doi: 10.7334/ psicothema2013.24

Parker, S. K. \& Bindl, U. (2017). Proactivity at work: A big picture perspective on a construct that matters. In S. K. Parker \& U. Bindl, U. (Eds.), Proactivity at work: Making things happen in organizations (pp. 1-20). New York, NY: Routledge.

Parker, S. K., \& Collins, C. (2010). Taking stock: Integrating and differentiating multiple proactive behaviours. Journal of Management, 36, 633-662. doi: $10.1177 / 0149206308321554$

Parker, S. K., Williamns, H., \& Turner, N. (2006). Modelling the antecedents of proactive behavior at work. Journal of Applied Psychology 91(3), 636-652. doi: 10.1037/00219010.91.3.636

Parker, S. K. \& Wang, Y. (2015). Helping people to 'make things happen': A framework for proactivity at work. International Coaching Psychology Review, 10, 62-75. 
Podsakoff. P. M., MacKenzie, S. B., \& Podsakoff, N. (2012). Sources of method bias in social science research and recommendations on how to control it. Annual Review of Psychology 63(1), 539-569. doi: 10.1146/annurev-psych-120710-100452

Podsakoff, P. M., MacKenzie, S. B., Paine, J. B., \& Bachrach, D. G. (2000). Organizational citizenship behaviours: A critical review of the theoretical and empirical literature and suggestions for future research. Journal of Management, 26(3), 513-563. doi: $10.1177 / 014920630002600307$

Salessi, S., \& Omar, A. (2017). Comportamientos proactivos en el trabajo: una revisión bibliográfica sistemática. Revista Argentina de Ciencias del Comportamiento 9(3), 82-103.

Salessi, S. \& Omar, A. (2016). Satisfacción laboral genérica. Propiedades psicométricas de una escala para medirla. Revista Alternativas en Psicología, 34, 93-108.

Schmitt, A., Den Hartog, D. N. \& Belschak, F. D. (2016). Transformational leadership and proactive work behaviour: A moderated mediation model including work engagement and job strain. Journal of Occupational \& Organizational Psychology, 89, 588-610. doi: 10.1111/joop.1214

Tabachnick, B. G., \& Fidell, L. S (2013). Using Multivariate Statistics (6th ed.). Boston, MA: Pearson.

Thomas, J., Whitman, D., \& Viswesvaran, C. (2010) Employee proactivity in organizations: A comparative meta-analysis of emergent proactive constructs. Journal of Occupational \& Organizational Psychology, 83, 275-300. doi: 10.1348/09637910X502359

Tornau, K. \& Frese, M. (2013). Construct clean-up in proactivity research: A metaanalysis on the nomological net of work-related proactivity concepts and their incremental validities. Applied Psychology: An International Review, 62, 44-96. doi: 10.1111/j.1464-0597.201200514.x

Ventura-León, J. \& Caycho, T. (2017). El coeficiente omega: un método alternativo para la estimación de la fiabilidad. Revista Latinoamérica de Ciencias Sociales, Niñezy Juventud, 15, 625-627.

Recibido: 28 de Setiembre 2017

Aceptado: 16 de Febrero 2018 